\title{
Budget cuts curb Europe's enthusiasm to revive innovation
}

Ambitious initiative to make Europe more competitive now limited in scope

\section{Cormac Sheridan}

Any attempt to stem Europe's slow decline in pharmaceutical innovation should be welcomed with open arms. At the beginning of the 1990s, six of the top ten medicines were developed in Europe, but within ten years this number dropped to only two. But cuts to the original budget for the European Commission's Seventh Framework

Programme (FP7) have inevitably led to a drastic slimming down of an ambitious, industry-led programme designed to revitalize the biopharmaceutical research environment in Europe.

The Innovative Medicines Initiative (IMI) aims to tackle some of the key bottlenecks in drug development by pooling resources from industry, academia, regulatory authorities and healthcare providers. The IMI, structured as a $50 / 50$ public-private partnership, was originally due to receive some $€ 440$ million per annum from the EC. Industry would have made a similar contribution in kind through its participation.

As a result of FP7's budget being cut from the intended $€ 72.7$ billion to an expected $€ 50.5$ billion for the period 2007-2013, the IMI is now expected to see its funding trimmed to around $€ 150-200$ million per annum, when averaged out over the lifetime of the programme, says Octavi Quintana-Trias, Director of Health Research within the EC's directorate general for research. The funding will also be back-loaded toward the end of the initiative. "We need some time to set up the

\section{Box 1 | Tackling bottlenecks}

The strategic research agenda for the Innovative Medicines Initiative identified four key bottlenecks in the drug development process:

- Improved prediction: early indications of safety

- Improved clinical performance: early indications of efficacy by use of biomarkers

- Better knowledge management: collaborating to break information barriers at the interfaces

- Bridging educational gaps: preclinical and clinical research and breaking barriers between disciplines

Source: Innovative Medicines Initiative website.

structure and start the operation and make the machine work," he says.

Not all of the agenda can be implemented following the cuts in the proposed budget, says Jonathan Knowles, Head of Global Research at Roche, and chairman of the research directors group of the European Federation of Pharmaceutical Industries and Associations (EFPIA), which is the driving force behind the IMI. But what will remain is still sufficiently large to have a substantial impact. "Much lower than that and I would start to have doubts," says Knowles. "This is already a compromise."

It has yet to be decided which of the IMI projects will stay and which will fall by the wayside. The initial strategic research

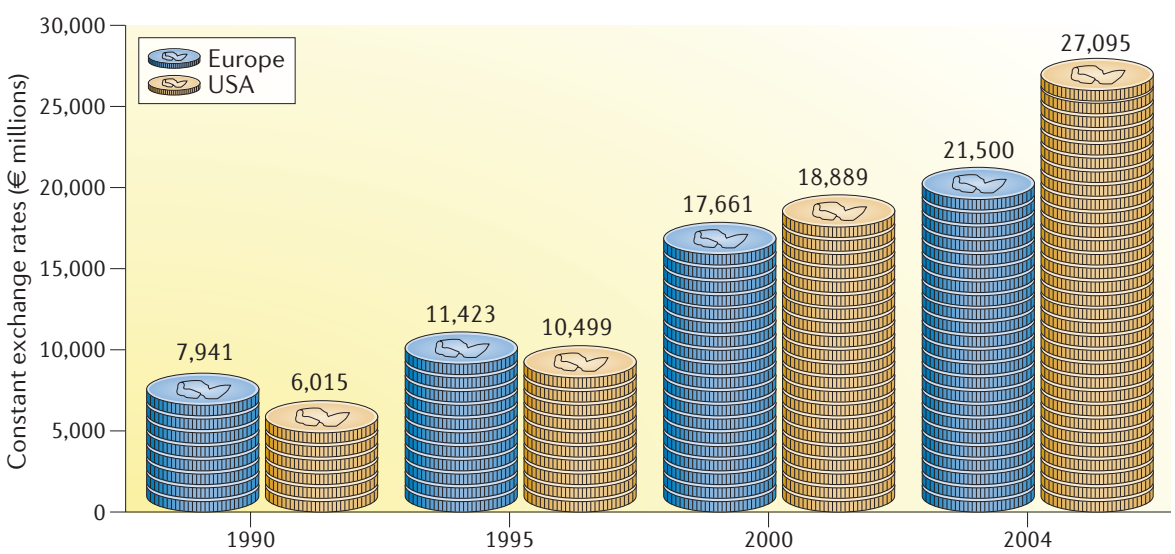

Europe has been struggling to compete with US investments in pharmaceutical RED. Source: EFPIA agenda, drawn up by stakeholders from industry, academia, regulatory agencies and patient groups in summer 2005, identified four main bottlenecks in drug development that urgently need addressing: improved prediction of safety; early indication of efficacy; knowledge management; and education and training (see BOX 1). This agenda will be applied across five disease areas - brain disorders, inflammatory disease, infectious disease, diabetes and cancer.

Roberto Solari, CEO of MRC Technology, the company responsible for commercializing research funded by the UK's Medical Research Council, says that the IMI should now focus on the safety and efficacy arms of the initiative, as these have the biggest short-term impact for pharma. "The knowledge management and education and training components are important but they are much longer term," says Solari, adding that establishing and funding experimental programmes in the areas of safety and efficacy will be easier than in the other two fields.

Aspects of safety and efficacy strategies in the IMI model are currently being tested in a Sixth Framework Programme pilot project. InnoMed, which has an €18-million budget and a 36-member consortium, is attempting to develop new diagnostics, as well as markers of disease progression and therapeutic response for Alzheimer's disease, and is also building a database that will supplement traditional toxicology information with data derived from 'omics' technologies.

Part of the objective of the toxicogenomics arm is to show how several companies can share information among themselves and with academic partners, an approach that Solari hopes IMI focuses on as it encourages greater mobility between academia and industry. "If the science base is really going to make an economic impact and get translated into industrial outcomes I believe we all need to get better at understanding one another and working together constructively," he says.

As is usual with the European Union, nothing will be fully agreed on until the many interested parties all reach consensus. FP7 is still crawling its way through a trilateral approval process that involves the EC, the European Parliament and the individual member states. FP7 is expected to be finally ratified in the autumn, with the IMl's first calls for proposals from scientists thought to follow shortly afterwards. For the many stakeholders who worry whether Europe will ever reclaim its position as a global leader in innovation, this opportunity to revive R\&D in Europe can't come a moment too soon. 